\title{
Finite Data Performance Analysis of MVDR Beamformer with and without Spatial Smoothing
}

Kalavai J. Raghunath, Student Member, IEEE, and V. Umapathi Reddy, Senior Member, IEEE

\begin{abstract}
Recently, the performance of a minimum variance distortionless response (MVDR) beamformer has been extensively studied for the case when a true or asymptotic covariance matrix is available. In practical situations, however, we only have a finite number of snapshots of data from which the array covariance matrix can be estimated. In this paper, we analyze the finite-data performance of this beamformer with and without spatial smoothing, using first-order perturbation theory. In particular, we develop expressions for the mean values of the power gain in any direction of interest, the output power and the norm of the weight-error vector, as a function of the number of snapshots and the number of smoothing steps. We show that, in general, the smoothing, in addition to decorrelating the sources, can also alleviate the effects of finite-data perturbations.

Next, we reduce the above expressions to the case when no spatial smoothing is used. These expressions are valid for an arbitrary array and for arbitrarily correlated signals. For this case, we also develop an expression for the variance of the power gain. We simplify these expressions for a single interference case to show explicitly how the SNR, spacing of the interference from the desired signal and the correlation between them influence the beamformer performance.

Simulations are used to verify the usefulness of the theoretical expressions and the results show an excellent agreement with predicted results.
\end{abstract}

\section{INTRODUCTION}

$I_{b}^{N}$ $\mathrm{N}$ a minimum variance distortionless response (MVDR) beamformer, the array weights are chosen so as to pass the desired directional (look direction) signal without any distortion while maximally rejecting the interfering signals. The only assumption made is that the desired signal direction is known a priori.

Since the pioneering work of Capon [6], there has been much activity in the development of optimum arrays for radar, sonar, communication, etc. Sensitivity of the adaptive arrays to element errors and to those in the look direction has been extensively studied in the recent past. Zahm [7] was one of the first to note the disastrous effects of pointing errors on the performance of adaptive arrays.

Manuscript received April 8, 1990; revised August 9, 1991.

K. J. Raghunath was with the Department of Electrical Communication Engineering, Indian Institute of Science, Bangalore 50012, India. He is now with the Department of Electrical Engineering, University of Minnesota, Minneapolis, MN.

V. U. Reddy is with the Department of Electrical Communication Engineering, Indian Institute of Science, Bangalore 560012, India.

IEEE Log Number 9202780
Cox [8] investigated the effects of mismatch in the look direction on the performance of two optimum beamformers, one based on the inversion of the noise alone crossspectral matrix and the other based on the inversion of signal-plus-noise cross-spectral matrix. Vural [9] studied the effects of system and medium perturbations (from assumed conditions) on the performance of adaptive arrays. Godara [10] presented an analysis of the effects of random errors in the weight vector and steering vector on the performance of two beamformers, the same as those considered by Cox.

This problem, i.e., sensitivity of the beamformer to errors of various kinds, was addressed by Hudson [11], Compton [12], and several others (see Godara [10] for more references), and more recently, by Jablon [13]. All these authors, however, do not address how the performance of the beamformer depends on the data size, i.e., the number of snapshots. The only papers (to our knowledge) which address this specific problem are those of Reed et al. [14] and Boroson [15]. Their analysis, however, assumes that the weight vector computed from one set of data operates on an independent set of data. Also, the main thrust of their analysis is directed to the case where the weight vector is estimated from the noise-alone matrix inverse, and the interferences are uncorrelated with the look-direction signal.

A factor which severely affects the performance of the MVDR beamformer even in the asymptotic case, is the correlation between the look-direction signal and the interferences. Not only does the beamformer fail to form deep nulls in the direction of the coherent interferences, but also the desired signal can be partially or fully cancelled in the output of the beamformer. This signal cancellation phenomenon in the presence of coherent interferences was recently analyzed by Reddy $\boldsymbol{e t}$ al. [4] and Paulraj et al. [17]. For the case of a uniform array, however, spatial smoothing has been proposed to alleviate the problems due to correlation [18]. In [4], Reddy et al. have shown that the effective correlation between the desireddirectional signal and the interfering signals decreases asymptotically with progressive smoothing thereby reducing the effects of correlation.

The analysis in [4] and [17] has been carried out for asymptotic case. In this paper, we analyze the performance of the MVDR beamformer in finite data with and 
without spatial smoothing. Our analysis uses first-order perturbation theory, originally developed by Wilkinson [3] and first applied in array processing problems by Kaveh and Barabell [1]. We derive expressions for the mean values of the power gain in any direction of interest, the output power, and the norm of the weight-error vector, as a function of the number of snapshots. We reduce these expressions to the case when no spatial smoothing is used. These expressions are valid for an array of arbitrary geometry with arbitrarily correlated impinging signals. For the unsmoothed case, we also develop an expression for the variance of the power gain. To get a better insight into the finite data effects, we simplify these expressions for the single interference case. Using these simplified expressions, we then show explicitly how the SNR, spacing of the interference from the desired signal, and the correlation between them influence the beamformer performance. In our analysis, unlike the case of Reed et al. [14] and Boroson [15], the weight vector is determined from the signal-plus-noise covariance matrix and is applied to the same data from which it is estimated.

Simulations are used to verify the usefulness of the theoretical expressions and the results show an excellent agreement with predicted results.

We state the problem in Section II and derive the expressions for the mean values of power gain, output power, and weight-error vector norm in Section III. The corresponding expressions for the unsmoothed case are in Section IV and the expression for the variance of the power gain is given in Section V. In Section VI, we present the simulation results, and finally in Section VII, we conclude the paper.

\section{Statement of the Problem}

Consider $D$ narrow-band sources impinging on an array consisting of $m$ elements. We assume that the $D$ signals $s_{1}(t), s_{2}(t), \cdots, s_{D}(t)$ are zero-mean, stationary, and ergodic complex Gaussian random processes. Let

$$
r^{T}(t)=\left[r_{1}(t), r_{2}(t), \cdots, r_{m}(t)\right]
$$

be the sampled vector (snapshot) of array signals (the superscript $T$ denotes transpose) and

$$
s^{T}(t)=\left[s_{1}(t), s_{2}(t), \cdots, s_{D}(t)\right]
$$

be the vector of source signals. Then

$$
r(t)=A s(t)+n(t)
$$

where $n(t)$ is the array noise vector, assumed to be zeromean, stationary, and ergodic complex Gaussian random process, independent of the signals, with covariance matrix given by $\sigma_{n}^{2} I$, where $\sigma_{n}^{2}$ is an unknown constant and $I$ is an identity matrix. $A$ is the $m$ by $D$ direction matrix

$$
A=\left[a_{1}, a_{2}, \cdots, a_{D}\right]
$$

where $a_{1}, a_{2}, \cdots, a_{D}$ are the direction vectors. The true or asymptotic array covariance matrix is then given by

$$
R=E\left[r(t) r^{+}(t)\right]
$$

where $(+)$ denotes Hermitian transpose.
Let $a_{1}$ represent the desired signal direction (look-direction) vector. The signals received at the sensors are combined to form the array output

$$
y(t)=w^{+} r(t)
$$

where $w$, the weight vector in MVDR beamformer, is determined such that the output power is minimized under the criterion that the gain in the look direction is unity. Thus

$$
w=\frac{R^{-1} a_{1}}{a_{1}^{+} R^{-1} a_{1}} .
$$

In spatial smoothing, we use a uniform linear array which is divided into $K$ subarrays, each of size $m$. The first subarray is formed from the sensors $\{1,2, \cdots, m\}$ and the second subarray from $\{2,3, \cdots, m+1\}$ and so on. Let $r_{i}(\cdot)$ denote the vector of received signals from the $i$ th subarray. Then the $i$ th subarray covariance matrix is given by

$$
R_{i}=E\left[r_{i}(t) r_{i}^{+}(t)\right]
$$

and the smoothed array covariance matrix is defined as

$$
\bar{R}=\frac{1}{K} \sum_{i=1}^{K} R_{i}
$$

We note here that subarray covariance matrices are the submatrices of the total array covariance matrix $R$ of size $M(=m+K-1)$ by $M$. For example, $R_{i}$ is the $m$-by- $m$ submatrix of $R$ with $R(i, i)$ as its first element. In the spatial smoothing case, the weight vector becomes

$$
\bar{w}=\frac{\bar{R}^{-1} a_{1}}{a_{1}^{+} \bar{R}^{-1} a_{1}} .
$$

In the finite data case, the array covariance matrix is estimated as

$$
\hat{R}=\frac{1}{L} \sum_{t=1}^{L} r(t) r^{+}(t)
$$

where $L$ is the number of snapshots. The corresponding smoothed array covariance matrix is given by

$$
\overline{\hat{R}}=\frac{1}{K} \sum_{i=1}^{K} \hat{R}_{i} .
$$

Again, $\hat{R}_{i}$ is a submatrix of $\hat{R}$ with $\hat{R}(i, i)$ as its first element. Note that all the terms corresponding to the finite data case are denoted by $(\hat{*})$. We should point out here that when spatial smoothing is not used, $L$ should at least be equal to $m$, since $\hat{R}$ (cf. (2.11)) would otherwise be rank deficient. On the other hand, with spatial smoothing, the minimum number of snapshots required (for $\hat{R}$ to attain full rank) can be traded with the number of subarrays $K$. For example, for $L=1, \overline{\hat{R}}$ would attain full rank if $K$ $\geq m$.

The weight vector with spatial smoothing is given by

$$
\overline{\hat{w}}=\frac{\overline{\hat{R}}^{-1} a_{1}}{a_{1}^{+} \overline{\hat{R}}^{-1} a_{1}} .
$$


The power gain and the output power can then be expressed as

$$
\overline{\hat{G}}_{j}=\overline{\hat{g}}_{j} \overline{\hat{g}}_{j}^{\star}
$$

with

$$
\overline{\hat{g}}_{j}=\frac{\boldsymbol{a}_{j}^{+} \overline{\hat{R}}^{-1} \boldsymbol{a}_{1}}{\boldsymbol{a}_{1}^{+} \overline{\hat{R}}^{-1} \boldsymbol{a}_{1}}
$$

and

$$
\overline{\hat{P}}_{\text {out }}=\frac{1}{a_{1}^{+} \overline{\hat{R}}^{-1} a_{1}} .
$$

When we refer these quantities, i.e., gain and output power, to the asymptotic case, we drop the symbol hat. Further, when we consider the unsmoothed case, i.e., $K$ $=1$, we drop the symbol overbar.

To study the deviation of the weight vector from its asymptotic value, $\bar{w}$, as a function of $L$, we define the weight-error vector norm as

$$
\frac{\|\overline{\hat{w}}-\bar{w}\|^{2}}{\|\bar{w}\|^{2}}
$$

where $\|\cdot\|$ denotes the Euclidean norm.

The problem is to evaluate the mean values $E\left[\bar{G}_{j}\right]$, $E\left[\hat{\hat{P}}_{\text {out }}\right]$ and $E\left[\|\overline{\hat{w}}-\bar{w}\|^{2} /\|\bar{w}\|^{2}\right]$ as a function of the number of snapshots.

III. Derivation of Expressions for $\boldsymbol{E}\left[\overline{\hat{G}}_{j}\right], \boldsymbol{E}\left[\overline{\hat{P}}_{\text {out }}\right]$ AND $E\left[\|\overline{\hat{w}}-\overline{\boldsymbol{w}}\|^{2} /\|\overline{\boldsymbol{w}}\|^{2}\right]$

The estimated array covariance matrix $\hat{R}$ can be decomposed as [1], [3]

$$
\hat{R}=R+\epsilon B
$$

where $R$ is the true or asymptotic covariance matrix, $B$ is a zero-mean random matrix, and $\epsilon$ is a perturbation constant. Since $\hat{R}_{i}$ is a submatrix of $\hat{R}$, we can write

$$
\hat{R}_{i}=R_{i}+\epsilon B_{i}
$$

where $B_{i}$ is the corresponding submatrix of $B$. It then follows that

$$
\overline{\hat{R}}=\bar{R}+\epsilon \bar{B}
$$

where $\bar{R}$ is as defined in (2.9) and $\bar{B}$ is given by

$$
\bar{B}=\frac{1}{K} \sum_{i=1}^{K} B_{i}
$$

We can now express

$$
\begin{aligned}
\overline{\hat{R}}^{-1}= & (\bar{R}+\epsilon \bar{B})^{-1}=\left\{I-\epsilon \bar{R}^{-1} \bar{B}+\epsilon^{2}(\bar{R}-1 \bar{B})^{2}\right. \\
& \left.-\epsilon^{3}\left(\bar{R}^{-1} \bar{B}\right)^{3}+\cdots\right\} \bar{R}^{-1} .
\end{aligned}
$$

When the number of snapshots is large, $\epsilon$ is a small quantity and, hence, we can neglect terms of order $\epsilon^{3}$ and higher and obtain results accurate to the first order in $L$. We thus approximate

$$
\overline{\hat{R}}^{-1} \approx\left\{I-\epsilon \bar{R}^{-1} \vec{B}+\epsilon^{2}\left(\bar{R}^{-1} \bar{B}\right)^{2}\right\} \bar{R}^{-1} .
$$

\section{A. Mean Power Gain}

Using (3.6) in (2.15) and dividing both the numerator and denominator by $a_{1}^{+} \bar{R}^{-1} a_{1}$, we obtain

$$
\overline{\hat{g}}_{j} \approx \frac{\bar{g}_{j}-\epsilon \delta_{3}+\epsilon^{2} \delta_{5}}{1-\epsilon \delta_{2}+\epsilon^{2} \delta_{4}}
$$

where

$$
\begin{aligned}
& \delta_{1}=\boldsymbol{a}_{1}^{+} \bar{R}^{-1} \boldsymbol{a}_{1} \\
& q_{1}=\bar{R}^{-1} \boldsymbol{a}_{1} \\
& q_{j}=\bar{R}^{-1} \boldsymbol{a}_{j} \\
& \delta_{2}=\frac{\boldsymbol{q}_{1}^{+} \bar{B} \boldsymbol{q}_{1}}{\delta_{1}} \\
& \delta_{3}=\frac{\boldsymbol{q}_{j}^{+} \overline{\boldsymbol{B}} \boldsymbol{q}_{1}}{\delta_{1}} \\
& \delta_{4}=\frac{q_{1}^{+} \overline{\boldsymbol{B}} \bar{R}^{-1} \overline{\boldsymbol{B}} \boldsymbol{q}_{1}}{\delta_{1}} \\
& \delta_{5}=\frac{\boldsymbol{q}_{j}^{+} \overline{\boldsymbol{B}} \bar{R}^{-1} \overline{\boldsymbol{B}} \boldsymbol{q}_{1}}{\delta_{1}} .
\end{aligned}
$$

Note that $\delta_{2}, \delta_{3}, \delta_{4}$, and $\delta_{5}$ are scalar random quantities while $\delta_{1}$ is deterministic. Combining (3.7) with (2.14) and neglecting the terms of order $\epsilon^{3}$ and higher, we obtain the following expression for the power gain in a direction with steering vector $\boldsymbol{a}_{j}$ :

$$
\overline{\hat{G}}_{j}=\overline{\hat{g}}_{j} \overline{\hat{g}}_{j}^{\star} \approx \frac{\bar{G}_{j}-2 \epsilon J_{1}+\epsilon^{2}\left(\delta_{3} \delta_{3}^{\star}+2 J_{2}\right)}{1-2 \epsilon \delta_{2}+\epsilon^{2}\left(\delta_{2}^{2}+2 \delta_{4}\right)}
$$

where

$$
\begin{aligned}
& J_{1}=\operatorname{Re}\left(\bar{g}_{j}^{\star} \delta_{3}\right) \\
& J_{2}=\operatorname{Re}\left(\bar{g}_{j}^{\star} \delta_{5}\right) .
\end{aligned}
$$

Using the approximation

$$
(1+y)^{-1} \approx 1-y+y^{2}
$$

for the denominator, combining the result with the numerator, and again neglecting the terms of order $\epsilon^{3}$ and higher, we get

$$
\begin{aligned}
\overline{\hat{G}}_{j} \approx & \bar{G}_{j}+\epsilon\left(2 \bar{G}_{j} \delta_{2}-2 J_{1}\right)+\epsilon^{2}\left(\left|\delta_{3}\right|^{2}-4 \delta_{2} J_{1}\right. \\
& \left.+3 \bar{G}_{j} \delta_{2}^{2}-2 \bar{G}_{j} \delta_{4}+2 J_{2}\right) .
\end{aligned}
$$

For any deterministic vectors $\alpha_{1}, \alpha_{2}, \alpha_{3}, \alpha_{4}$ of appropriate dimension, we have

$$
E\left[\alpha_{1}^{+} \bar{B} \alpha_{2}\right]=0
$$

since $B$ is zero mean and the matrix $\bar{B}$ is just a sum of submatrices of $B$, and (as shown in Appendix A)

$$
\begin{aligned}
& E\left[\alpha_{1}^{+} \bar{B} \alpha_{2} \alpha_{3}^{+} \bar{B} \alpha_{4}\right] \\
& \quad=\frac{1}{\epsilon^{2} K^{2} L} \sum_{u=1}^{K} \sum_{v^{\prime}=1}^{K} \alpha_{1}^{+} R_{u v} \alpha_{4} \alpha_{3}^{+} R_{v^{\prime} u} \alpha_{2}
\end{aligned}
$$


where $R_{u v}$ denotes the submatrix of size $m$ by $m$ of total array covariance matrix $R$ beginning at element $R(u, v)$. Note that the subarray matrix $R_{i}$ is the same as $R_{i i}$. Following the approach outlined in Appendix B, we can evaluate the expectation of each term in (3.12) and obtain

$$
\begin{aligned}
E\left[\overline{\hat{G}}_{j}\right] \approx & \bar{G}_{j}+\frac{1}{K^{2} L \delta_{1}^{2}} \sum_{u=1}^{K} \sum_{v=1}^{K} \\
& \cdot\left\{\phi_{j j} \phi_{11}^{\star}-4 \operatorname{Re}\left(\bar{g}_{j}^{\star} \phi_{j 1} \phi_{11}^{\star}\right)+3 \bar{G}_{j} \phi_{11} \phi_{11}^{\star}\right. \\
& \left.-2 \bar{G}_{j} \phi_{11} T_{1} \delta_{1}+2 \operatorname{Re}\left(\bar{g}_{j}^{\star} \phi_{j 1} T_{1} \delta_{1}\right)\right\}
\end{aligned}
$$

where

$$
\begin{aligned}
\phi_{x y} & =q_{x}^{+} R_{u v} q_{y} \\
T_{1} & =\operatorname{tr}\left(\bar{R}^{-1} R_{v u}\right) .
\end{aligned}
$$

Since $a_{j}$ is arbitrary, (3.15) can be used to find the power gain in any direction and thus obtain the mean power beam pattern of the beamformer for finite data.

\section{B. Mean Output Power}

Following the approach used above, we can obtain

$$
\overline{\hat{P}}_{\mathrm{out}} \approx \frac{1}{\delta_{1}}\left\{1+\epsilon \delta_{2}+\epsilon^{2}\left(\delta_{2}^{2}-\delta_{4}\right)\right\}
$$

Using (3.13) and (3.14), we can show that

$$
E\left[\overline{\hat{P}}_{\text {out }}\right] \approx \bar{P}_{\text {out }}\left\{1+\frac{1}{K^{2} L} \sum_{u=1}^{K} \sum_{v=1}^{K}\left(\frac{\phi_{11} \phi_{11}^{\star}}{\delta_{1}^{2}}-\frac{\phi_{11} T_{1}}{\delta_{1}}\right)\right\}
$$

\section{Mean Weight-Error Vector Norm}

Using the approximations (3.6) and (3.11) and the definition (2.13), we can show that

$$
\frac{\|\overline{\hat{w}}-\bar{w}\|^{2}}{\|\bar{w}\|^{2}} \approx \frac{\epsilon^{2}}{x_{1}}\left(x_{3}+x_{1} \delta_{2}^{2}-\delta_{2} x_{2}\right)
$$

where

$$
\begin{aligned}
& x_{1}=q_{1}^{+} q_{1} \\
& x_{2}=2 \operatorname{Re}\left(q_{1}^{+} \bar{R}^{-1} \bar{B} q_{1}\right) \\
& x_{3}=q_{1}^{+} \bar{B} \bar{R}^{-1} \bar{R}^{-1} \bar{B} q_{1} .
\end{aligned}
$$

Following the approach outlined in Appendix B, we can evaluate the expectation of each term in $(3.19)$ and obtain

$$
\begin{aligned}
E\left[\frac{\|\overline{\hat{w}}-\bar{w}\|^{2}}{\|\bar{w}\|^{2}}\right] \approx & \frac{1}{K^{2} L} \sum_{u=1}^{K} \sum_{v=1}^{K} \\
& \cdot\left\{\frac{\phi_{11} T_{2}}{x_{1}}+\frac{\left|\phi_{11}\right|^{2}}{\delta_{1}^{2}}-\frac{\tau}{\delta_{1} x_{1}}\right\}
\end{aligned}
$$

where

$$
\begin{aligned}
T_{2} & =\operatorname{tr}\left(R_{v u} \bar{R}^{-1} \bar{R}^{-1}\right) \\
\tau & =2 \operatorname{Re}\left(\phi_{11} q_{1}^{+} \bar{R}^{-1} R_{v u} q_{1}\right) .
\end{aligned}
$$

Note that the RHS in (3.21) goes to zero as $L$ tends to infinity, which is consistent with the fact that $\overline{\hat{w}}$ tends to $\bar{w}$ asymptotically.

\section{EXPRESSIONS for UnSMOOTHEd CASE}

In this section, we reduce the above results to the unsmoothed case. It should, however, be noted that even though the above results were derived for the case of a uniform linear array, the following results are valid for an arbitrary array.

For the unsmoothed case, i.e., $K=1$, we have

$$
\begin{aligned}
\phi_{11} & =\delta_{1} \\
\phi_{j 1} & =g_{j} \delta_{1} \\
\phi_{j j} & =a_{j}^{+} R^{-1} a_{j} \\
T_{1} & =m .
\end{aligned}
$$

Substituting (4.1) into (3.15) gives

$$
E\left[\hat{G}_{j}\right] \approx G_{j}\left(1-\frac{1}{L}\right)+\frac{Q_{j}}{L}
$$

where

$$
Q_{j}=\frac{\boldsymbol{a}_{j}^{+} \boldsymbol{R}^{-1} \boldsymbol{a}_{j}}{\delta_{1}}
$$

Similarly, (3.18) reduces to

$$
E\left[\hat{P}_{\text {out }}\right] \approx P_{\text {out }}\left(1-\frac{m-1}{L}\right) .
$$

Note from (4.4) that the mean value of the output power is within $3 \mathrm{~dB}$ of the asymptotic value when the number of snapshots $L$ is approximately twice the array size $m$.

The weight-error vector norm for the unsmoothed case similarly reduces to

$$
E\left[\frac{\|\hat{w}-w\|^{2}}{\|w\|^{2}}\right] \approx \frac{1}{L}\left(\frac{\delta_{1} \operatorname{tr}\left(R^{-1}\right)}{x_{1}}-1\right) .
$$

We now study the finite data performance of the beamformer in certain special, but pertinent, scenarios. We do this by considering a single interference.

\section{A. Single Interference Case}

For the case of a single interference, we can write

$$
\begin{aligned}
R= & \sigma_{n}^{2}\left(\boldsymbol{I}+\frac{\sigma_{1}^{2}}{\sigma_{n}^{2}} a_{1} a_{1}^{+}+\frac{\sigma_{2}^{2}}{\sigma_{n}^{2}} a_{2} a_{2}^{+}+\frac{\sigma_{1} \sigma_{2}}{\sigma_{n}^{2}} \rho a_{1} a_{2}^{+}\right. \\
& \left.+\frac{\sigma_{1} \sigma_{2}}{\sigma_{n}^{2}} \rho^{\star} a_{2} a_{1}^{+}\right)
\end{aligned}
$$


where $\sigma_{1}^{2}, \sigma_{2}^{2}$, and $\sigma_{n}^{2}$ are the powers of the look-directional signal, interference (jammer) and noise, respectively, $\rho$ is the coefficient of correlation between the signal and the jammer, and $a_{2}$ is the jammer direction vector. Applying the matrix inversion lemma repeatedly to (4.6), we can express (4.3) as

$$
Q_{2}=\frac{\boldsymbol{a}_{2}^{+} R^{-1} \boldsymbol{a}_{2}}{\boldsymbol{a}_{1}^{+} R^{-1} \boldsymbol{a}_{1}}=\frac{\sigma_{1}^{2}\left(m^{2}-|\beta|^{2}\right)+m \sigma_{n}^{2}}{\sigma_{2}^{2}\left(m^{2}-|\beta|^{2}\right)+m \sigma_{n}^{2}}
$$

where $\beta=\boldsymbol{a}_{2}^{+} \boldsymbol{a}_{1}$. Substituting (4.7) and the expression for the gain in the interference direction (given in [4]) in (4.2), we get

$$
\begin{aligned}
E\left[\hat{G}_{2}\right] \approx & \frac{\psi \psi^{\star}}{\left\{\sigma_{2}^{2}\left(m^{2}-|\beta|^{2}\right)+m \sigma_{n}^{2}\right\}^{2}}\left(1-\frac{1}{L}\right) \\
& +\frac{\sigma_{1}^{2}\left(m^{2}-|\beta|^{2}\right)+m \sigma_{n}^{2}}{L\left\{\sigma_{2}^{2}\left(m^{2}-|\beta|^{2}\right)+m \sigma_{n}^{2}\right\}}
\end{aligned}
$$

where

$$
\psi=\sigma_{1} \sigma_{2} \rho^{\star}\left(|\beta|^{2}-m^{2}\right)+\sigma_{n}^{2} \beta .
$$

We will now simplify (4.8) for some special scenarios.

1) Low Noise Case $\left(\sigma_{n}^{2}<<\sigma_{1}^{2}, \sigma_{2}^{2}\right)$ : When the noise is low enough such that $m\left(\sigma_{i}^{2} / \sigma_{n}^{2}\right)\left(1-|\beta|^{2} / m^{2}\right) \gg 1$ for $i=1$ and 2 , (4.8) can be simplified to

$$
E\left[\hat{G}_{2}\right] \approx \frac{\sigma_{1}^{2}}{\sigma_{2}^{2}}\left(|\rho|^{2}+\frac{1-|\rho|^{2}}{L}\right) .
$$

Note from (4.10) that when the signals are uncorrelated $(\rho=0)$, the gain varies as $1 / L$ and thus, for a $20 \mathrm{~dB}$ suppression of the jammer power below the signal power, we require about 100 snapshots. When signals are correlated, (4.10) shows that the degradation in the interference rejection performance due to finite data is overshadowed by that due to correlation. For example, for $\rho=$ 0.5 , the reduction in the jammer power goes up by a mere $0.1 \mathrm{~dB}$ when $L$ is increased from 100 to infinity.

We now consider the mean output power given by (4.4). Assuming once again that $m\left(\sigma_{i}^{2} / \sigma_{n}^{2}\right)\left(1-|\beta|^{2} / m^{2}\right)>$ 1 for $i=1$ and 2,

$$
E\left[\hat{P}_{\text {out }}\right] \approx \sigma_{1}^{2}\left(1-|\rho|^{2}\right)\left(1-\frac{m-1}{L}\right)
$$

where we used the simplified result for $P_{\text {out }}$ from [4]. Note from (4.11) that in addition to signal cancellation due to correlation, a reduction in output power also takes place because of finite data.

2) High Noise Power $\left(\sigma_{n}^{2} \gg \sigma_{1}^{2}, \sigma_{2}^{2}\right)$ : From (4.8), we can write

$$
E\left[\hat{G}_{2}\right] \approx \frac{|\beta|^{2}}{m^{2}}+\frac{1}{L}\left(1-\frac{|\beta|^{2}}{m^{2}}\right) .
$$

Thus, there exists high enough noise such that the finite data power gain is independent of the correlation and the signal powers, and depends solely on the separation of the sources. We may point out here that this expression reduces to the result given in [4] in the asymptotic case, i.e., $L=\infty$.

3) Jammer Close to Look Direction $\left(|\beta|^{2} \approx m^{2}\right)$ : When the jammer is close to the look direction, $|\beta|$ approaches $m$ and (4.8) simplifies to

$$
E\left[\hat{G}_{2}\right] \approx \frac{|\beta|^{2}}{m^{2}} .
$$

Thus, the power gain is independent of $L$ and the MVDR beamformer reduces to a conventional beamformer, as in the asymptotic case (see [4]).

\section{Variance of Power Gain in Unsmoothed Case}

In the previous section, we obtained the expression for mean power gain. But, how close this expression predicts the result in any given experiment depends on its variance, defined as

$$
\operatorname{var}\left(\hat{G}_{j}\right)=E\left[\hat{G}_{j}^{2}\right]-\left\{E\left[\hat{G}_{j}\right]\right\}^{2} .
$$

Using the earlier approximations, i.e., neglecting the terms of order $\epsilon^{3}$ and above, and deriving an expression for the variance, it has been found that the result is not accurate enough. Hence, to get a better approximation, we retain terms up to $\epsilon^{4}$ and obtain a result accurate to second order in $L$. Thus, we use the approximations

$$
\begin{aligned}
\hat{R}^{-1} \approx & \left\{I-\epsilon R^{-1} B+\epsilon^{2}\left(R^{-1} B\right)^{2}\right. \\
& \left.-\epsilon^{3}\left(R^{-1} B\right)^{3}+\epsilon^{4}\left(R^{-1} B\right)^{4}\right\} R^{-1}
\end{aligned}
$$

and

$$
(1+y)^{-1} \approx\left\{1-y+y^{2}-y^{3}+y^{4}\right\}
$$

and carry out the analysis, retaining terms upto $\epsilon^{4}$ at every stage. While taking expectations, in addition to identities of the type (3.13) and (3.14) for the unsmoothed case, we also use the following identities, given in [16]:

$$
\begin{aligned}
& E\left[\alpha_{1}^{+} B \alpha_{2} \alpha_{3}^{+} B \alpha_{4} \alpha_{5}^{+} B \alpha_{6}\right] \\
& =\frac{1}{L^{2} \epsilon^{3}}\left\{\alpha_{1}^{+} R \alpha_{4} \alpha_{3}^{+} R \alpha_{6} \alpha_{5}^{+} R \alpha_{2}\right. \\
& \left.\quad+\alpha_{1}^{+} R \alpha_{6} \alpha_{3}^{+} R \alpha_{2} \alpha_{5}^{+} R \alpha_{4}\right\}
\end{aligned}
$$

and

$$
\begin{aligned}
& E\left[\alpha_{1}^{+} B \alpha_{2} \alpha_{3}^{+} B \alpha_{4} \alpha_{5}^{+} B \alpha_{6} \alpha_{7}^{+} B \alpha_{8}\right] \\
& =\frac{1}{L^{2} \epsilon^{3}}\left\{\alpha_{1}^{+} R \alpha_{4} \alpha_{3}^{+} R \alpha_{2} \alpha_{5}^{+} R \alpha_{8} \alpha_{7}^{+} R \alpha_{6}\right. \\
& \quad+\alpha_{1}^{+} R \alpha_{6} \alpha_{3}^{+} R \alpha_{8} \alpha_{5}^{+} R \alpha_{2} \alpha_{7}^{+} R \alpha_{4} \\
& \left.\quad+\alpha_{1}^{+} R \alpha_{8} \alpha_{3}^{+} R \alpha_{6} \alpha_{5}^{+} R \alpha_{4} \alpha_{7}^{+} R \alpha_{2}\right\}
\end{aligned}
$$

Note here that $\alpha_{1}$ to $\alpha_{8}$ are determinisitic vectors of appropriate dimension. The detailed steps involved in evaluating the terms in RHS of (5.1) are given in [5] and the 
final results are as follows:

$$
\begin{aligned}
E\left[\hat{G}_{j}^{2}\right] \approx & G_{j}^{2}+\frac{4 G_{j}\left(Q_{j}-G_{j}\right)}{L} \\
& +\frac{G_{j}^{2}(6-2 m)+G_{j} Q_{j}(2 m-8)+2 Q_{j}^{2}}{L^{2}}
\end{aligned}
$$

and

$$
\begin{aligned}
E\left[\tilde{G}_{j}\right] \approx & G_{j}\left(1-\frac{1}{L}\right)+\frac{Q_{j}}{L} \\
& +\frac{G_{j}(5+3 m)+Q_{j}(m-1)}{L^{2}} .
\end{aligned}
$$

The variance is then obtained by substituting (5.6) and (5.7) into (5.1) and retaining terms up to $1 / L^{2}$ :

$$
\begin{aligned}
\operatorname{var}\left(\hat{G}_{j}\right) \approx & \frac{2 G_{j}\left(Q_{j}-G_{j}\right)}{L} \\
& +\frac{G_{j}^{2}(-5-8 m)-8 G_{j} Q_{j}+Q_{j}^{2}}{L^{2}} .
\end{aligned}
$$

Note that the variance goes to zero as $L$ tends to infinity implying that the estimate $\hat{G}_{j}$ is consistent.

We may point out here that the first two terms in (5.7) are identical to those of (4.2) and the third term is the result due to the inclusion of $\epsilon^{3}$ and $\epsilon^{4}$ terms in the approximations. Clearly, (5.7) predicts more accurately than (4.2) when the number of snapshots is small.

The variance expression is quite unwieldy and does not explicitly show the magnitude of the quantity involved. Hence, it would be useful to simplify this expression for some scenarios. From (4.2), we see that the degradation due to finite data depends on the relative values of $G_{j}$ and $Q_{j} / L$. For the case of uncorrelated interferences that are widely spaced from the desired signal, the asymptotic gain $G_{j}$ is very low and hence finite data gain is essentially controlled by $Q_{j} / L$. For example, for the case of very low noise [4], $G_{j} \approx 0$. Thus, in such cases, $Q_{j} / L \gg G_{j}$. Using this approximation and assuming that $L$ is much larger than $m,(5.7)$ and (5.8) can be simplified as

$$
E\left[\hat{G}_{j}\right] \approx \frac{Q_{j}}{L}
$$

and

$$
\operatorname{var}\left[\hat{G}_{j}\right] \approx \frac{Q_{j}^{2}}{L^{2}}
$$

Thus the variance is approximately equal to the square of the mean in such scenarios.

\section{Simulation Results and Discussions}

We conducted simulations to test the usefulness of the expressions derived in the earlier sections. In our simulations, a uniform linear array with a half wavelength in- terelement spacing was used, though the expressions for the unsmoothed case are valid for an arbitrary array. In all the scenarios, the desired signal was assumed to arrive from the broadside direction $\left(0^{\circ}\right)$. We generated a snapshot vector of data $r(t)$ (cf. (2.3)) by generating a vector $\boldsymbol{n}(t)$ consisting of $m$ zero-mean, unit variance, independent complex Gaussian random variables, and another vector $s(t)$ consisting of two zero-mean, complex Gaussian random variables whose variances were fixed to give the desired individual signal powers. In the case of uncorrelated interference the two random variables comprising the signal vector were generated independently. In the case of correlated interference, we applied Cholesky decomposition to the 2 by 2 covariance matrix with desired correlation, and used the Cholesky factor so obtained to transform a vector of 2 uncorrelated complex Gaussian random variables to give the required signal vector. We repeated this to generate independent snapshots of data, changing the seeds randomly from snapshot to snapshot. This whole procedure was repeated to generate an independent set of snapshots in each Monte Carlo run. The simulated result was obtained by averaging over 50 Monte Carlo runs. The particulars of the number of sensors, DOA's and powers of the sources, noise power and correlation between the look-direction signal and the inteference, are described in the figures.

We first considered the case with spatial smoothing, keeping the total number of elements in the array at 9. Fig. 1 shows the mean power gain $(E[\hat{G}])$ in the interferer direction for different number of smoothing steps. Fig. 1(a) gives the results for the case when the interference is fully correlated with the look-direction signal, while the results of Fig. 1(b) correspond to the uncorrelated case. The results show that there is an excellent agreement between the theoretical and the experimental results. In the case of fully correlated interference (Fig. 1(a)), we see that the performance improves with more number of subarrays $(K)$ even at the expense of reduced subarray size $(m)$ with consequent reduction in the effective aperture. This performance improvement is mainly due to the reduction in the effective correlation between the impinging signals. Note that for $K=6$, when sources are sufficiently decorrelated, we can observe a change in the gain with the number of snapshots. This is consistent with our earlier comments (see Section IV-A1) that when the correlation between the look-direction signal and the interference is high, the degradation in the gain due to correlation is so large that the effect of $L$ (number of snapshots) on the gain performance is hardly noticed.

In the asymptotic case, trading for more number of subarrays at the expense of reduced effective aperture is not beneficial when the sources are uncorrelated. But in the finite data case, there is a significant improvement in the gain with increasing number of subarrays even at the expense of decreasing subarray size for any given number of snapshots (see Fig. 1(b)). For example, if we need a $40 \mathrm{~dB}$ suppression in the interference direction, we require about 150 snapshots with no smoothing, i.e., with 


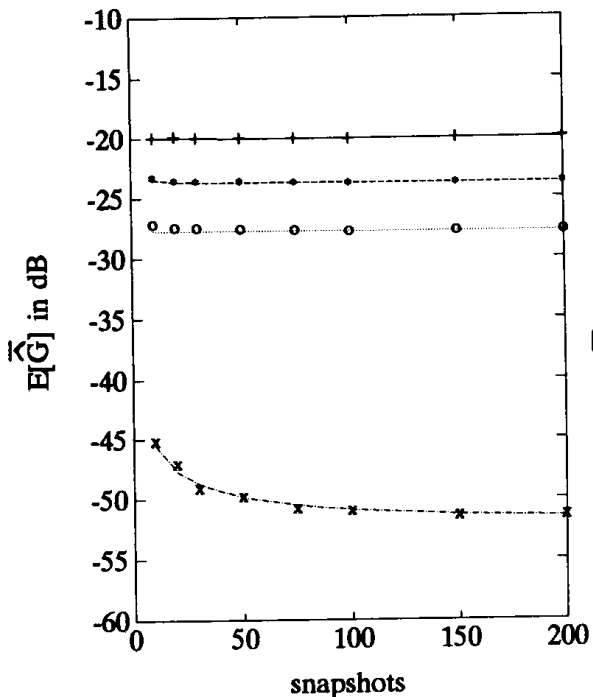

(a)

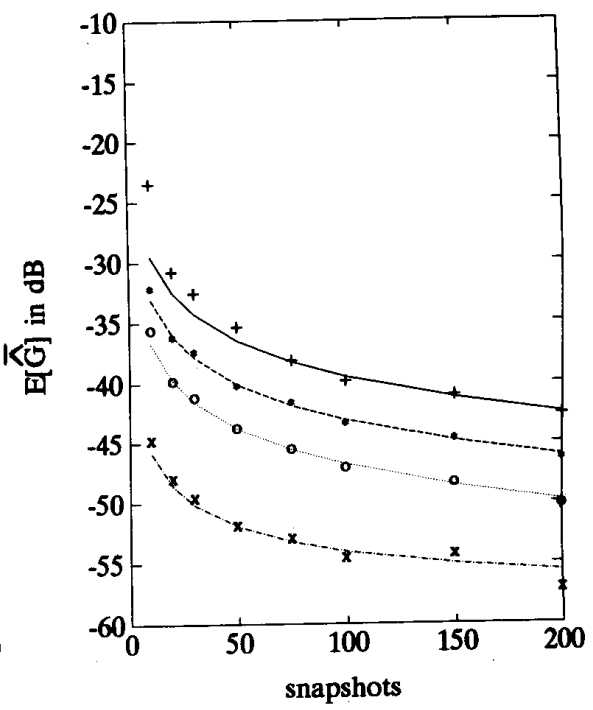

(b)

Fig. 1. Mean power gain in the inteferer direction in the presence of a single interference for different number of subarrays, $K$ Fig. 1. Mean pow $=1,3,4,6$, with the total number of elements fixed at 9 (DOA's $=0,20$, powers ", simulation " $\star$ " ; for $K=4$, theoretical $=0^{\circ}$; for $K=1$ theoretical " $\_$," simulation " +"'; for $K=3$, theoretical " ${ }^{\prime}$ "). (a) Fully correlated interference, $\rho=1$. (b) "..." simulation " $O$ "; for $K=6$

all the nine elements in one single array, while about 60 snapshots are enough when we use smoothing with 3 subarrays, with 7 elements in each subarray. If we further increase the smoothing by one more subarray (decreasing the subarray size to 6 ), the required number of snapshots comes down to 20 . These results convey that smoothing is preferable to a single larger array, at least for the signal scenario considered here. This improvement in the performance with smoothing is because of the reduction in the finite data perturbations as also noted in [19].

The source spacing, considered in Fig. 1 , is relatively large as it is about $4 / 3$ rd and $1 / 2$ of the effective beamwidths of the subarrays corresponding to $K=1$ and $K=$ 6 , respectively. To see how this tradeoff between the number of subarrays and the subarray size affects the performance in the scenarios with different source spacings, particularly in the case of uncorrelated interference, we have evaluated (3.15) for various combinations of $K$ and $m$, keeping the total number of elements at 8 , for different angular separations between the interference and the lookdirection signal, and plotted the results in Fig. 2. As in Fig. 1, we considered two cases, one with fully correlated interference and the other with uncorrelated interference, for two different snapshot sizes ( $L=20$ and $L=200)$ in each case. For the case when the interference is fully correlated with the look-direction signal (Figs. 2(a) and (c)), we can see that the performance improves with more subarrays even at the expense of decreasing the subarray size with consequent reduction in the effective aperture. The effect is more pronounced in the case of larger angular separation of the interference (see plots 3 and 4), as was the case in Fig. 1. This is due to the fact that smoothing decorrelates faster when sources are widely separated. Note that the behavior is not affected by the number of snapshots. In the presence of uncorrelated interference, trading for more subarrays at the expense of decreasing the subarray size is still beneficial, in general, except when the angular separation between the interference and the look-direction signal is too small (Figs. 2(b) and (d)). When the sources are very close, the effective aperture becomes an important factor and hence the performance is dictated by this (see plots 1 and 2 of Fig. 2(b)). When the snapshots are more, the finite data perturbations are smaller, and hence, the aperture effect becomes more important, causing the performance to degrade for very small subarray sizes even at larger angular separation (see 3 and 4 of Fig. 2(d)). Since, in practice, we do not know whether the interference is correlated or not, the results of Fig. 2 seem to favor more smoothing at the expense of reduced subarray size.

Fig. 3 shows the finite-data behavior of the mean output power of the beamformer and the mean weight-error vector norm for the case of a fully correlated interference, for two different smoothing steps, i.e., $K=2$ and $K=6$. With more smoothing, the effective correlation between the desired signal and the interference comes down thereby resulting in a reduced signal cancellation. Consequently, the output power goes up, as seen in Fig. 3(b). 


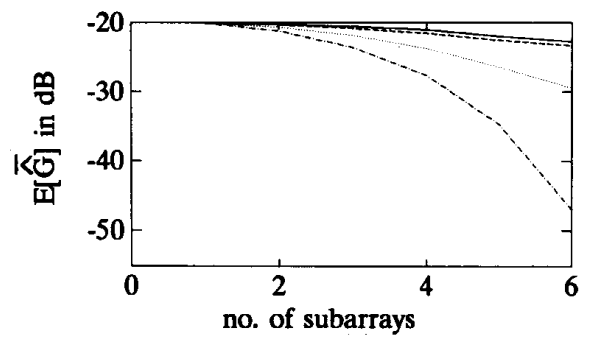

(a)

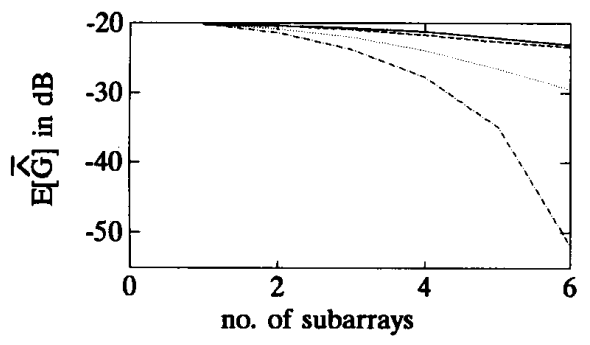

(c)

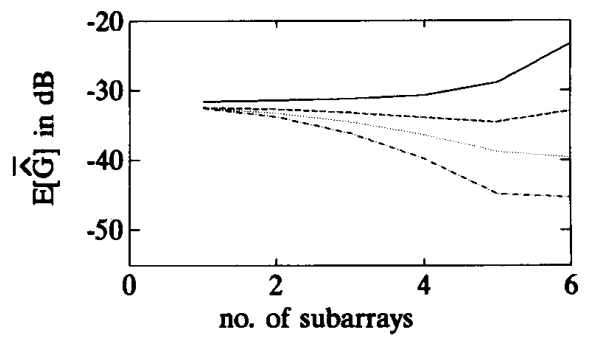

(b)

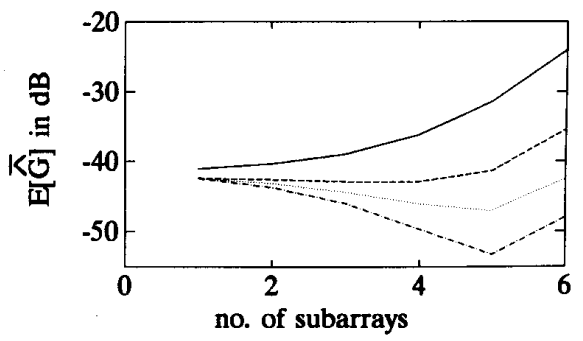

(d)

Fig. 2. Mean power gain in the interferer direction under a tradeoff between the number of subarrays and subarray size for different angular separations between the look-direction signal and the interference, and for two different snapshot sizes (total

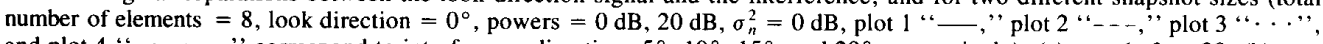
and plot 4 "“- $-\cdot-\cdot$, , correspond to interference directions $5^{\circ}, 10^{\circ}, 15^{\circ}$, and $20^{\circ}$, respectively). (a) $\rho=1, L=20$. (b) $\rho=$ $0, L=20$. (c) $\rho=1, L=200$. (d) $\rho=0, L=200$.

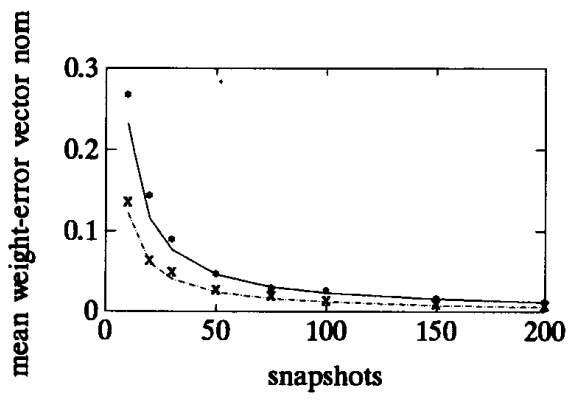

(a)

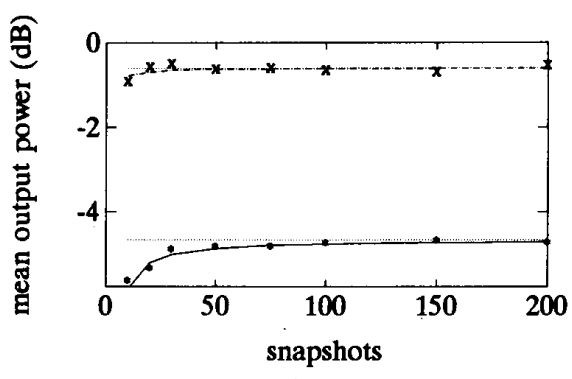

(b)

Fig. 3. Finite data behavior for two different smoothing steps, $K=2$ and $K=6$, in the presence of a fully correlated interference $\left(m=6\right.$, DOA's $=0^{\circ}, 10^{\circ}$, powers $=0 \mathrm{~dB}, 20 \mathrm{~dB}, \sigma_{n}^{2}=0 \mathrm{~dB}, \rho=1$, look direction $=0^{\circ}$; for $K=2$, theoretical " - ," simulation " $\star$ "; for $K=6$, theoretical "......", simulation " $\times$ "; asymptotic ....."). (a) Mean weight-error vector norm. (b) Mean output power of the beamformer.

Recall further that progressive smoothing results in progressive reduction in the finite data perturbations, and as a result, the finite data weight vector is nearer to its asymptotic value when $K$ is larger (see Fig. 3(a)). Because of this, the output power is closer to the asymptotic value in the case with $K=6$ compared to that with $K=$ 2 (see Fig. 3(b)).

We next considered the case with no smoothing. In this case, we first considered the effect of correlation on the finite data performance and the results are shown in Fig. 4. As in the previous cases, theoretical predictions are very close to the simulated. From the simplified expressions for the case of a single interference with low sensor noise (cf. (4.10)), it was noted that when correlation is high, the degradation due to correlation overshadows the degradation due to finite data. Fig. 4(a) shows that this is in fact generally true. For $\rho=0.5$ and $\rho=0.9$, the gain does not change with the number of snapshots $L$ at all, while for $\rho=0.2$ and more so for $\rho=0$, the effect of $L$ is quite pronounced. The convergence rate of the mean output power to the asymptotic value is not dependent on the correlation, as predicted by the expression (4.4). 


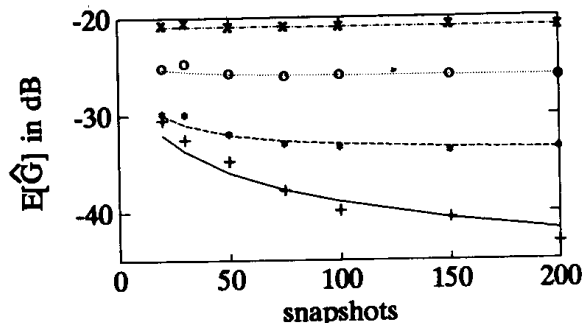

(a)

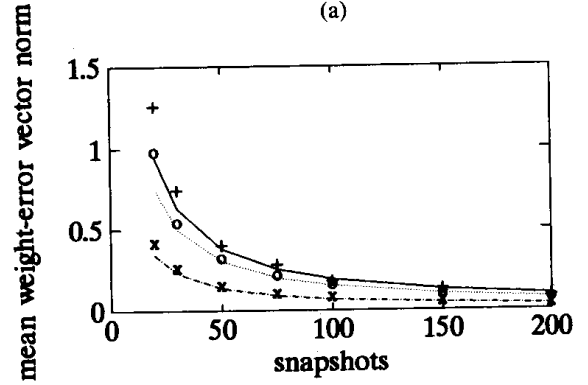

(c)

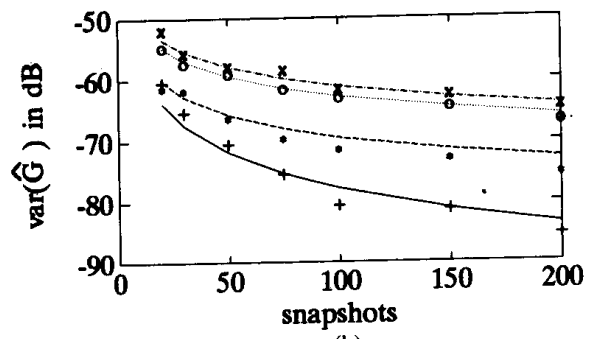

(b)

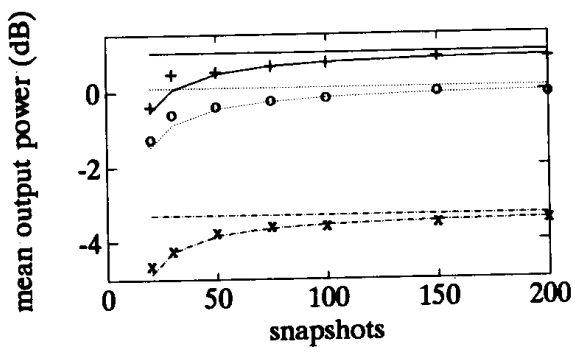

(d)

Fig. 4. Finite data performance curves for the case of a single correlated interference without spatial smoothing ( $m=6$, DOA's $=0^{\circ}, 10^{\circ}$, powers $=0 \mathrm{~dB}, 20 \mathrm{~dB}, \sigma_{n}^{2}=0 \mathrm{~dB}$, look direction $=0^{\circ}$; for $\rho=0.9$, theoretical "..- - , " simulation, " $\times$, ,", APG $=-21.0 \mathrm{~dB}$; for $\rho=0.5$, theoretical " ...,", simulation " 0, , APG $=-26.1 \mathrm{~dB}$; for $\rho=0.2$, theoretical ",--- ," simulation " $\star, "$ APG $=-34.1 \mathrm{~dB}$; for $\rho=0$, theoretical "__," simulation "+", APG $=-55.6 \mathrm{~dB}$; APG $=$ asymptotic simulation " $\star, " A P G=-34.1 \mathrm{~dB}$; for $\rho=0$, theoretical (bain). (a) Mean power gain in the interferer direction. (b) Variance of the power gain in the interferer direction. (c) Mean weight-error vector norm. (d) Mean output power of the beamformer.

In Section III, we had made a comment that the gain expression can be used to find the beam power pattern for finite data. In the earlier examples we had considered only the power gain in the interferer direction. Here, we plotted the finite data power beam patterns for two different snapshot sizes, $L=20$ and 200 (see Fig. 5). The asymptotic beam power pattern is also shown for reference. We observe a good agreement between experimental and theoretical results. Further, the gain in the neighborhood of the interference direction reaches very close to the aysmptotic value even at $L=20$ (see Fig. 5), which is consistent with the result that the gain in the direction of the correlated interference changes very little with the number of snapshots. However, the improvement with higher number of snapshots is clearly seen at other directions.

Fig. 6 shows the power gain in the interferer direction as a function of the look-direction signal power for two levels of interference power, $-10 \mathrm{~dB}$ and $10 \mathrm{~dB}$, with the noise level fixed at $0 \mathrm{~dB}$. The number of snapshots was fixed at 30 , i.e., $L=30$. The plots show that the theoretical results predict quite accurately the gain performance for the whole range of signal and interference powers. Note that the asymptotic values do not change with the look-direction signal power, as predicted by (4.8) (substitute $L=\infty$ and $\rho=0$ ). But for finite data, the expression (4.8) and the plots show that the gain performance degrades with increasing look-direction signal power. This is a result of signal corruption, a fact also noted by Reed et al. [14] and Boroson [15]. As in the

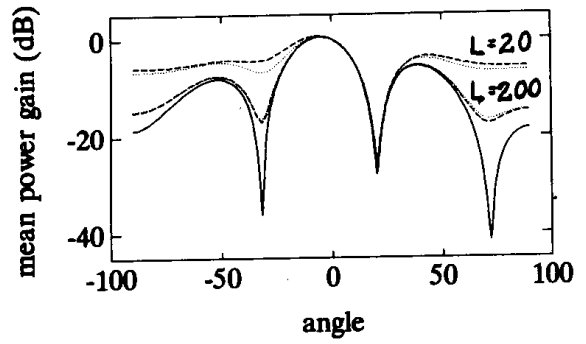

Fig. 5. Finite data power beam patterns in the presence of a single correlated infererence $\left(m=4\right.$, DOA's $=0^{\circ}, 20^{\circ}$, powers $=0 \mathrm{~dB}, 20 \mathrm{~dB}, \sigma_{n}^{2}$ $=0 \mathrm{~dB}, \rho=0.4$, look-direction $=0^{\circ}$; theoretical “...," simulation ".--,," asymptotic "___,").

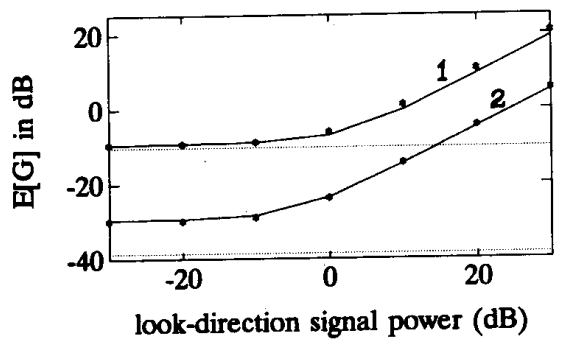

Fig. 6. Mean power gain in the interferer direction for the case of a single uncorrelated interference as a function of look-direction signal power ( $m$ $=4, \mathrm{DOA}^{\prime} \mathrm{s}=0^{\circ}, 20^{\circ}, L=30, \sigma_{n}^{2}=0 \mathrm{~dB}, \rho=0$, look direction $=0^{\circ}$; plots 1 and 2 correspond to interference powers of $-10 \mathrm{~dB}$ and $+10 \mathrm{~dB}$, respectively; theoretical "___," simulation " $\star$, ," asymptotic "...."). 
asymptotic case, the null depth in the interferer direction goes up with increasing interference power.

\section{Conclusions}

In this paper, we have analyzed the performance of the MVDR beamformer with and without spatial smoothing, using first-order perturbation theory. We have developed expressions for the mean power gain in any direction of interest, the mean output power and the mean weight-error vector norm as a function of the number of snapshots. While the expressions for the spatially smoothed case are valid only for an uniform linear array, those for the case with no smoothing are valid for arbitrary array. No assumptions have been made regarding the number of sources or the correlation between them. The expressions for the unsmoothed case are simplified for the two-source case to explicitly show the effect of signal powers, spacing between the desired and interfering signals and the correlation between them.

Our analysis shows that for uncorrelated sources, the performance degradation from the asymptotic value is dependent on the data size, while in the correlated case, the degradation due to finite data is overshadowed by that due to correlation. The results for the spatially smoothed case (with the total number of elements fixed) indicate that in addition to decorrelating the sources, the smoothing can also reduce the perturbation effects caused by finite data. Since in a practical scenario, it may not be known a priori whether the interferences are correlated or not, use of more smoothing at the expense of reduced effective aperture, in general, seems to be favorable. This will result in decorrelation of the sources mainly when correlated interferences are present, and reduction in finite-data perturbations when the interferences are uncorrelated. It should, however, be noted that the performance may degrade at very low subarray sizes (due to aperture effect) when the interference is very close to the look-direction signal and is uncorrelated with it.

\section{APPEndix A}

In this Appendix, we derive the identity (3.14). We can write this as

$$
\begin{aligned}
E\left[\alpha_{1}^{+} \bar{B} \alpha_{2} \alpha_{3}^{+} \bar{B} \alpha_{4}\right]= & E\left[\sum_{n=1}^{K} \sum_{t=1}^{K} \alpha_{1}(n)^{\star} \bar{B}(n, t) \alpha_{2}(t)\right. \\
& \left.\cdot \sum_{y=1}^{K} \sum_{z=1}^{K} \alpha_{3}(y) \star \bar{B}(y, z) \alpha_{4}(z)\right] \\
= & \sum_{n=1}^{K} \sum_{t=1}^{K} \sum_{y=1}^{K} \sum_{z=1}^{K} \alpha_{1}(n)^{\star} \alpha_{2}(t) \\
& \cdot \alpha_{3}(y)^{\star} \alpha_{4}(z) E[\bar{B}(n, t) \bar{B}(y, z)] .
\end{aligned}
$$

We can express each element of the smoothed matrix $\bar{B}$ in terms of the elements of the overall matrix $B$ as

$$
\bar{B}(n, t)=\frac{1}{K} \sum_{u=1}^{K} B(n+u-1, t+u-1) .
$$

We can then write

$$
\begin{aligned}
& E[\bar{B}(n, t) \bar{B}(y, z)] \\
& =\frac{1}{K^{2}} \sum_{u=1}^{K} \sum_{v=1}^{K} E[B(n+u-1, t+u-1) \\
& \quad \cdot B(y+v-1, z+v-1)] .
\end{aligned}
$$

Using the results from [2], we can show that

$$
E[B(w, x) B(y, z)]=\frac{R(w, z) R(y, x)}{L \epsilon^{2}} .
$$

Substituting (A.4) into (A.3) gives

$$
\begin{aligned}
E[\bar{B}(n, t) \bar{B}(y, z)] & \\
= & \frac{1}{K^{2} L \epsilon^{2}} \sum_{u=1}^{K} \sum_{v=1}^{K} R(n+u-1, z+v-1) \\
& \cdot R(y+v-1, t+u-1) .
\end{aligned}
$$

Using (A.5) in (A.1), and simplifying, we get

$E\left[\alpha_{1}^{+} \bar{B} \alpha_{2} \alpha_{3}^{+} \bar{B} \alpha_{4}\right]=\frac{1}{\epsilon^{2} K^{2} L} \sum_{u=1}^{K} \sum_{v=1}^{K} \alpha_{1}^{+} R_{u v} \alpha_{4} \alpha_{3}^{+} R_{v u} \alpha_{2}$

where $R_{u v}$ is the $m$ by $m$ submatrix of the total array matrix $R$ beginning at the element $R(u, v)$.

\section{APPENDIX B}

In this Appendix, we outline the method for evaluating the expectation of the terms in (3.12) and (3.19) using the identities (3.13) and (3.14).

In view of the identity (3.13), the expectation of the terms associated with $\epsilon$ go to zero. Now, consider a term associated with $\epsilon^{2}$, say $\delta_{4}$. From the definition of $\delta_{4}$

$$
E\left[\delta_{4}\right]=E\left[\frac{q_{1}^{+} \bar{B} \bar{R}^{-1} \bar{B} q_{1}}{\delta_{1}}\right] .
$$

Using spectral decomposition

$$
\bar{R}^{-1}=\sum_{i=1}^{m} \frac{1}{\mu_{i}} \gamma_{i} \gamma_{i}^{+}
$$

in (B.1) and interchanging the order of expectation and summation, we get

$$
E\left[\delta_{4}\right]=\frac{1}{\delta_{1}} \sum_{i=1}^{m} \frac{1}{\mu_{i}} E\left[\boldsymbol{q}_{1}^{+} \bar{B} \gamma_{i} \gamma_{i}^{+} \bar{B} \boldsymbol{q}_{1}\right]
$$

which, in view of (3.14), simplifies to

$$
E\left[\delta_{4}\right]=\frac{1}{\delta_{1} K^{2} L \epsilon^{2}} \sum_{i=1}^{m} \frac{1}{\mu_{i}} \sum_{u=1}^{K} \sum_{v=1}^{K} \boldsymbol{q}_{1}^{+} R_{u v} \boldsymbol{q}_{1} \gamma_{i}^{+} R_{v u} \gamma_{i}
$$


Since $\gamma_{i}^{+} R_{v u} \gamma_{i}$ is a scalar, we can write

$$
E\left[\delta_{4}\right]=\frac{1}{\delta_{1} K^{2} L \epsilon^{2}} \sum_{i=1}^{n} \frac{1}{\mu_{i}} \sum_{u=1}^{K} \sum_{v=1}^{K} q_{1}^{+} R_{u v} q_{1} \operatorname{tr}\left(\gamma_{i} \gamma_{i}^{+} R_{v u}\right)
$$

where $\operatorname{tr}(\cdot)$ denotes the trace operator. Now taking the summation over $i$ inside the trace operator and using (B.2), we finally obtain

$$
E\left[\delta_{4}\right]=\frac{1}{\delta_{1}^{2} K^{2} L \epsilon^{2}} \sum_{u=1}^{K} \sum_{v^{\prime}=1}^{K} \phi_{11} T_{1}
$$

where

$$
\begin{aligned}
\phi_{11} & =\boldsymbol{q}_{1}^{+} \boldsymbol{R}_{u \prime} \boldsymbol{q}_{1} \\
T_{1} & =\operatorname{tr}\left(\bar{R}^{-1} R_{v u}\right) .
\end{aligned}
$$

Similarly, we can find the expectation of the other terms in (3.12) and (3.19).

\section{ACKNOWLEDGMENT}

The authors would like to thank the referees for their very careful reading of the manuscript and their many useful suggestions, which greatly increased the quality and readability of this paper.

\section{REFERENCES}

[1] M. Kaveh and A. J. Barabell, "The statistical performance of the MUSIC and minimum-norm algorithms," IEEE Trans. Acoust., Speech, Signal Processing, vol. 34, pp. 331-341, Apr. 1986.

[2] D. R. Brillinger, Time Series: Data Analysis and Theory. New York: Holt, Rinehart and Winston, 1975.

[3] J. H. Wilkinson, The Algebraic Eigenvalue Problem. New York: Oxford University Press, 1965.

[4] V. U. Reddy, A. Paulraj, and T. Kailath, "Performance analysis of the optimum beamformer in the presence of correlated sources and its behavior under spatial smoothing," IEEE Trans. Acoust., Speech, Signal Processing, vol. ASSP-35, pp. 927-936, July 1987.

[5] K. J. Raghunath, "Performance analysis of MVDR beamformer in finite data: With and without spatial smoothing," M.Sc.(Eng) thesis, Dep. Elec. Commun. Eng., Indian Institute Sci., Mar. 1990.

[6] J. Capon, "High-resolution frequency-wavenumber spectrum analysis," Proc. IEEE, vol. 57. pp. 1408-1418, Aug. 1969.

[7] C. L. Zahm, "Effects of the errors in the direction of incidence on the performance of an adaptive array," Proc. IEEE, vol. 60, no. 8, pp. 1008-1009, Aug. 1972.

[8] H. Cox, "Resolving power and sensitivity to mismatch of optimum array processors," J. Acoust. Soc. Amer., pp. 771-785, vol. 50, Sept. 1973.

[9] A. M. Vural, "Effects of perturbations on the performance of optimum/adaptive arrays," IEEE Trans. Aerosp. Electron. Syst., vol. AES-15, pp. 76-87, Jan. 1979.

[10] L. C. Godara, "Error analysis of the optimal antenna array processors," IEEE Trans. Aerosp. Electron. Syst., vol. AES-22, pp. 395 409, July 1986.

[11] J. E. Hudson, Adaptive Array Principles. New York: Peter Peregrinus and IEEE, 1981.
[12] R. T. Compton, Jr., "Pointing accuracy and dynamic range in a steered beam array,' Trans. Aerosp. Electron. Syst., vol. AES-16, pp. 280-287, May 1980.

[13] N. K. Jablon, "Adaptive beamforming with imperfect arrays," $\mathrm{Ph} . \mathrm{D}$. dissertation, Dep. Elec. Eng., Stanford Univ., Aug. 1985.

[14] L. S. Reed, J. D. Mallet, and L. E. Brennan, "Rapid convergence rate in adaptive arrays," IEEE Trans. Aerosp. Electron. Syst., vol. AES-10, pp. 853-863, Nov. 1974.

[15] D. M. Boroson, "Sample size considerations for adaptive arrays," IEEE Trans. Aerosp. Electron. Syst., vol. AES-16, pp. 446-451, July 1980.

[16] D. R. Farrier, D. J. Jeffries, and R. Mardani, "Theoretical performance prediction of the MUSIC algorithm," Proc. Inst. Elec. Eng. vol. 135, pt. F, no. 3, pp. 216-224, June 1988.

[17] A. Paulraj, V. U. Reddy, and T. Kailath, "Analysis of signal cancellation due to multipath in optimum beamformers for moving arrays," IEEEJ. Ocean. Eng., vol. OE-12, pp. 163-172, Jan. 1987.

[18] T. J. Shan and T. Kailath, "Adaptive beamforming for coherent signals and interferences," IEEE Trans. Acoust., Speech, Signal Processing, vol. ASSP-33, pp. 527-536, June 1985.

[19] A. Paulraj, V. U. Reddy, T. J. Shan, and T. Kailath, "Performance analysis of the MUSIC algorithm with spatial smoothing in the presence of coherent sources," in Proc. IEEE MILCOM Conf. (Monterey, CA), Oct. 1986.

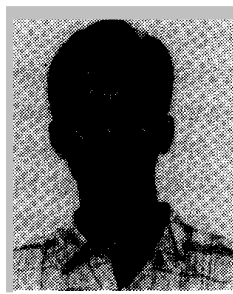

Kalavai J. Raghunath (S'87) received the B.E. degree in electronics and communication engineering from Osmania University, Hyderabad, India, in 1988, and the M.S. (Engg.) degree in electrical communication engineering from the Indian Institute of Science, Bangalore, India, in 1990. Currently, he is working towards the Ph.D. degree in electrical engineering at the University of Minnesota, Minneapolis. His research interests are in the areas of VLSI algorithms and architectures for signal processing, neural networks, and statis-

tical signal processing.

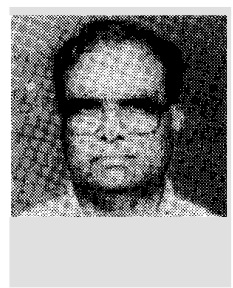

V. Umapathi Reddy (S'66-M'70-SM'82) received the B.E. and M.Tech degrees in electronics and communication engineering, from Osmania University and the Indian Institute of Technology (IIT), Kharagpur, in 1962 and 1963, respectively, and $\mathrm{Ph} . \mathrm{D}$. degree in electrical engineering from the University of Missouri in 1971.

He was an Assistant Professor at IIT, Madras, during 1972-1976 and Professor at IIT, Kharagpur, during 1976-1979. During 1979-1982, he was a Visiting Professor at the Department of Electrical Engineering, Stanford University. In April 1982, he joined Osmania University as a Professor, and was the Founder-Director of the Research and Training Unit for Navigational Electronics, funded by the Department of Electronics, Government of India. Since April 1988, he has been with the Indian Institute of Science, Bangalore, as a Professor of Electrical Communication Engineering. He has served as a consultant in signal processing to Avionics Design Bureau of Hindustan Aeronautics Limited, Hyderabad, and to Central Research Laboratory, Bharat Electronics Ltd., Bangalore. His recent research interests are in sensitivity analysis, adaptive algorithms, adaptive arrays, and neural networks.

Dr. Reddy is a Fellow of the Indian Academy of Sciences and Indian National Academy of Engineering, and Fellow of the Institution of Electronics and Telecommunications Engineers (IETE), India. He received the S. K. Mitra Memorial Award (1989) from IETE for the best research paper. 